\title{
The Effect of Grid Misalignment on the Uniformity of Response of Gas Proportional Scintillation Counters ${ }^{+}$
}

\author{
A.C.S.S.M. Bento, J.M.F. dos Santos and R.E.Morgado ${ }^{1}$ \\ Departamento de Física, Universidade de Coimbra, P-3000 Coimbra, PORTUGAL \\ 'Los Alamos National Laboratory, Los Alamos, New Mexico 87545, USA
}

\section{Abstract}

In the present work we revisit the simplifying assumptions of an earlier theoretical treatment on the effects of grid misalignment on the performance of a planar gas proportional scintillation counter (GPSC). We present new experimental and computational results to establish the dependence of the effects of misalignment in terms of the reduced electric field in the scintillation region, the finite size of the electron cloud, and the solid angle subtended by the photosensor. It is shown that solid angle effects will offset to some degree the effects of grid misalignment in an optimized detector. For the reduced fields normally used in the scintillation region of an optimized GPSC $\left(5 \mathrm{~V} \mathrm{~cm}^{-1}\right.$ torr $\left.{ }^{-1}\right)$, misalignments as large as $3 \%$ have less than a $2 \%$ effect on pulse-amplitude variations. This effect is less than the statistical fluctuations in the number of primary electrons nominally produced in the absorption of $\mathrm{x}$-rays in the 1- to 6-keV energy region. For reduced fields closer to the scintillation threshold $\left(1 \mathrm{Vcm}^{-}\right.$ ${ }^{1}$ torr $\left.{ }^{-1}\right), 15 \%$ variations in pulse amplitudes are possible, although it is unlikely that a GPSC would be operated under such unfavorable conditions.

\section{INTRODUCTION}

The gas proportional scintillation counter (GPSC) [1-3] is the radiation sensor of choice in many applications: $x$-ray astronomy, energy-dispersive $\mathrm{x}$-ray fluorescence analysis, and Mossbauer spectroscopy. The GPSC is distinguished operationally from conventional proportional counters by the manner in which the primary ionization is amplified. In the GPSC, amplification is accomplished by the scintillation process in a well-localized region of the detector, whereas the proportional counter derives its amplification from the charge avalanche process. This difference results in reduced statistical fluctuations and, consequently, improved energy resolution in the GPSC.

When the filling gas is one atmosphere of high-purity xenon, the GPSC is a mechanically simple, rugged, compact and efficient detector of $x$-rays in the 1- to $60-\mathrm{keV}$ range. In conjunction with a photomultiplier tube (PMT) to record the scintillation light pulses, the GPSC delivers excellent energy resolution (less than $8 \%$ FWHM at $6 \mathrm{keV}$, compared to $15 \%$ for proportional counters) and good linearity over the full range of $x$-ray energies $[4,5]$, with the simplicity of room-

+ The authors acknowledge travel support from Fundação Luso Americana para o Desenvolvimento (FLAD, Lisboa), and Fundação Calouste Gulbenkian (Lisboa). temperature operation.

An important advantage that the GPSC has over roomtemperature solid state detectors, such as $\mathrm{CdZnTe}$ or $\mathrm{HgI}_{2}$, is its potentially large active area [5-7], a prerequisite for applications requiring high sensitivity. However, the energy resolution of a large-area GPSC is degraded by several factors which have been identified and examined by others [8-11]. In the present work, we will report on another factor that affects the performance of large-area GPSCs, namely, the effects due to misalignments of the scintillation grids.

The accuracy of grid alignment in a GPSC depends on the mechanical tolerances achieved during the fabrication and assembly of detector components. Acceptable alignment can be a demanding requirement in high-pressure detectors where grid spacings are typically a few millimeters. In all cases it is important to quantify the effects of grid misalignment in order to determine the mechanical tolerances required to achieve a given design performance and to suggest alternative measures to compensate for the misalignment effects.

\section{RATIONALE}

For the planar GPSC design shown in Figure 1, detector performance depends, in part, on the uniformity of the electric field in the scintillation region. A misalignment of the parallel grids in this region will affect the uniformity of response to the extent that the electric field is perturbed.

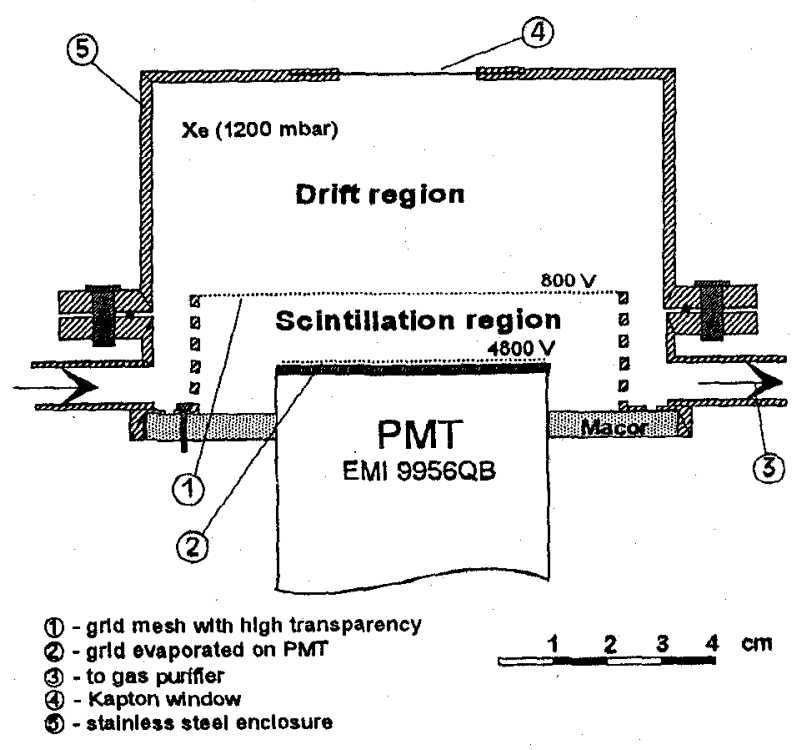

Figure 1: The planar GPSC schematic. 
This problem was first examined in [12] as a variation in the scintillation output as a function of the reduced electric field, $E / p$, where $E$ is the electric field between the grids and $p$ is the gas pressure. The conclusion reached then was that the effect was significant only for values of $E / p$ near the scintillation threshold, although no experimental results were presented to support this conclusion.

We now revisit the simplifying assumptions of the earlier theoretical work and present experimental and computational data to quantify the magnitude of the effect of grid misalignment on the performance of a planar GPSC. The results are analyzed and compared with the conclusions of [12].

\section{DESCRIPTION}

In the electric field of the scintillation region, the primary electrons gain enough energy between collisions to excite the gas atoms whose subsequent de-excitation results in the emission of a large number of ultraviolet (UV) scintillation photons. The dependence of the scintillation yield, $Y$, in photons per electron per centimeter, on the electric field between the scintillation grids has been studied both experimentally and theoretically [12-14]. The reduced yield, $\mathrm{Y} / \mathrm{p}$, as a function of the reduced electric field, $\mathrm{E} / \mathrm{p}$, in the scintillation region, is depicted in Figure 2. The reduced yield behaves approximately linearly with $\mathrm{E} / \mathrm{p}$, with a threshold near $(\mathrm{E} / \mathrm{p})_{0}=1 \mathrm{~V}-\mathrm{cm}^{-1}$-torr ${ }^{-1}$.

The experimental data for the reduced yield, $Y / p$, presented in Figure 2, were obtained with a well-aligned detector (GPSC-2, described below) and represents the centroid of the pulse-height distribution as a function of $\mathrm{E} / \mathrm{p}$ in the scintillation region for a $1-\mathrm{mm}$ collimated $5.9-\mathrm{keV} \mathrm{x}-$ ray beam along the detector central axis.

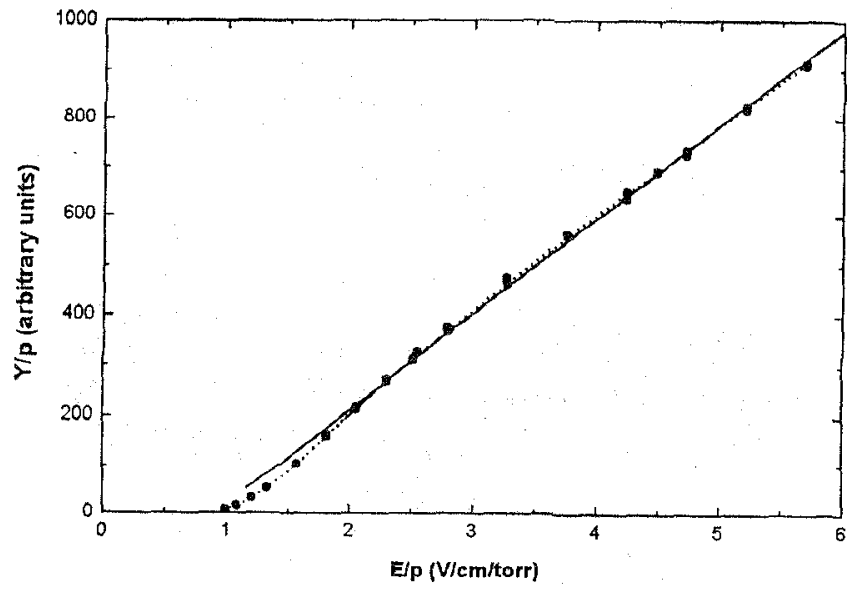

Figure 2: The reduced scintillation yield, $\mathrm{Y} / \mathrm{p}$, in xenon as a function of the reduced electric field, $\mathrm{E} / \mathrm{p}$.

Below this threshold, the mean kinetic energy of the electrons between collisions is insufficient to excite scintillation in xenon. This feature is exploited in the drift region where the reduced field is chosen well below the scintillation threshold so that detector response is independent of the position at which the $\mathrm{x}$-ray is absorbed. Above $6 \mathrm{Vcm}^{-1}$ torr ${ }^{-1}$ the ionization threshold is exceeded and the resolution of the GPSC is degraded by the large statistical fluctuations inherent in the charge multiplication process. For optimum GPSC performance, the reduced field in the scintillation region is selected for maximum light output, but below the ionization threshold.

For a planar GPSC, the average number of photons produced per electron crossing the scintillation region, $\mathrm{N}$, is given by

$$
\mathrm{N}=a\left[\mathrm{~V}-\left(\frac{\mathrm{E}}{\mathrm{p}}\right)_{0} \mathrm{px}\right]
$$

where $\mathrm{V}$ is the voltage difference between grids, $\mathrm{x}$ is the distance between grids, and $a$ is a constant.

For grid misalignments of the type shown in Figure 3, the variation in the number of scintillation photons, $\Delta N / N$, due to grid spacing variations, $\Delta \mathrm{x}$, can be shown to be approximately [12]

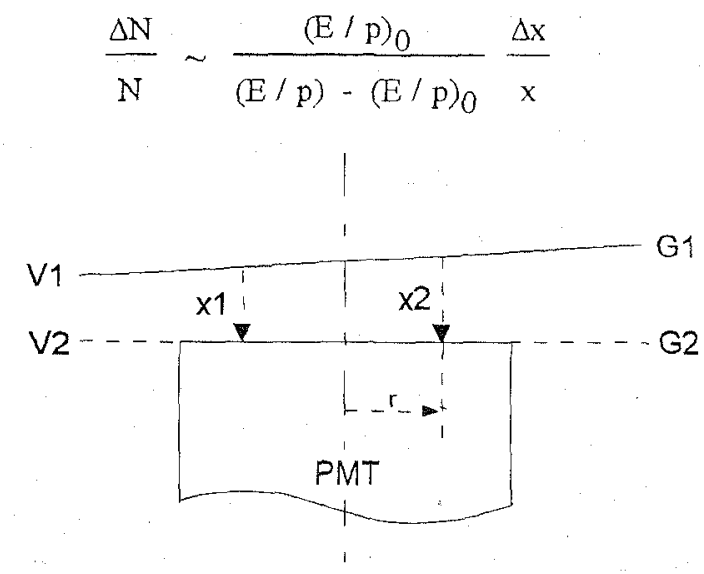

Figure 3: Grid misalignument schematic of a planar GPSC

For a planar GPSC with cylindrical symmetry, the effect of the misalignment would be manifest as a variation in pulse amplitude as a function of the azimuthal angle for a fixed value of the radius, $r$, from the detector axis.

However, Equation (2) is an approximation. First of all, the dependence of $\mathrm{N}$ on $\mathrm{E} / \mathrm{p}$ is not strictly linear over its entire range, but has a slower decrease as $E / p$ approaches the scintillation threshold (Figure 2). Secondly, the finite size and the effects of both lateral and longitudinal diffusion of the primary electron cloud entering the scintillation region have been ignored. Thirdly, the solid angle subtended by the photocathode varies with grid spacing and has also been ignored in the simplifying assumptions of Equation (2). Thus, the actual response of a GPSC detector with a misaligned grid would be expected to be different from the response predicted by Equation (2). To determine the effect 
of grid misalignments on GPSC performance, experimental measurements and computer simulations were performed for two different GPSC designs.

\section{EXPERIMENTAL RESULTS}

A $1-\mathrm{mm}$ collimated beam of $5.9-\mathrm{keV} \mathrm{x}$-rays was used to generate pulse-height distributions as a function of $\mathrm{E} / \mathrm{p}$ (quoted at the detector axis) in two different planar GPSC detectors. GPSC-1 had grid-spacing misalignments of the order of $1 \mathrm{~mm}$ at a radial distance of $35 \mathrm{~mm}$, while GPSC-2 had a maximum misalignment error of less than $0.1 \mathrm{~mm}$, at a radial distance of $35 \mathrm{~mm}$. GPSC-1 and GPSC-2 were described in detail in [15] and [16]. Measurements were performed for different azimuthal angles, $\phi$, at a fixed radial distance, $r=10 \mathrm{~mm}$, from the cylindrical axis of the detector. At this radius, grid-spacing misalignment is about $0.3 \mathrm{~mm}$ (3\%) for GPSC-1.

The measured pulse-height distributions were fitted to a gaussian superimposed on a linear background using the GRIDLS method [17]. The centroid position, A, of the pulseheight distributions for each detector is shown in Figures 4a and $4 \mathrm{~b}$ as a function of $\phi$ for selected values of $E / p$. The data is normalized to the azimuthal direction $\phi=0$, which was chosen to be perpendicular to the dynode slats of the venetianblind type photomultiplier tube (PMT). Error bars are shown only for the extreme values of $\mathrm{E} / \mathrm{p}$ and are due mainly to the uncertainty in the positioning of the 1-mm collimated beam. The large variation in the $\phi$-response in the data of both figures is a well-documented $[8,10,18]$ artifact of the venetian-blind type of PMT that was used in both detectors. Although the effect of the PMT asymmetry dominates the performance characteristics, the salient features of grid misalignment can still be easily distinguished.

In Figure 4a for GPSC-1, the dependence of the scintillation yield on $E / p$ is only significant near the scintillation threshold, as predicted by Equation (2). However, since the variations in the centroid position depicted in Fig. 4a are due to both PMT nonuniformity and grid misalignment effects, it is more illustrative to separate their respective contributions.

For $E / p$ values of $5 \mathrm{~V}-\mathrm{cm}^{-1}-$ torr ${ }^{-1}$, variations in the centroid position due to grid misalignment are about $1 \%$ [12] while variations due to PMT nonuniformity are of the order of $15 \%$, which are independent of $E / p$. By normalizing the centroid position variations for the different $E / p$ values to $\mathrm{E} / \mathrm{p}=5 \mathrm{~V}-\mathrm{cm}^{-1}-$ torr $^{-1}$, we can separate out the contribution due to grid misalignment for each value of $E / p$. The normalized centroid-position variations depicted in Figure 5 were obtained from Figure $4 \mathrm{a}$ by dividing the values for each $\mathrm{E} / \mathrm{p}$ by the corresponding values obtained for $5 \mathrm{~V} \mathrm{~cm}^{-1}$-torr ${ }^{-1}$, then normalizing to the maximum value obtained.

Fig. 5 shows that, for each $E / p$ value, variations in the centroid position of the pulse-height distributions due to grid
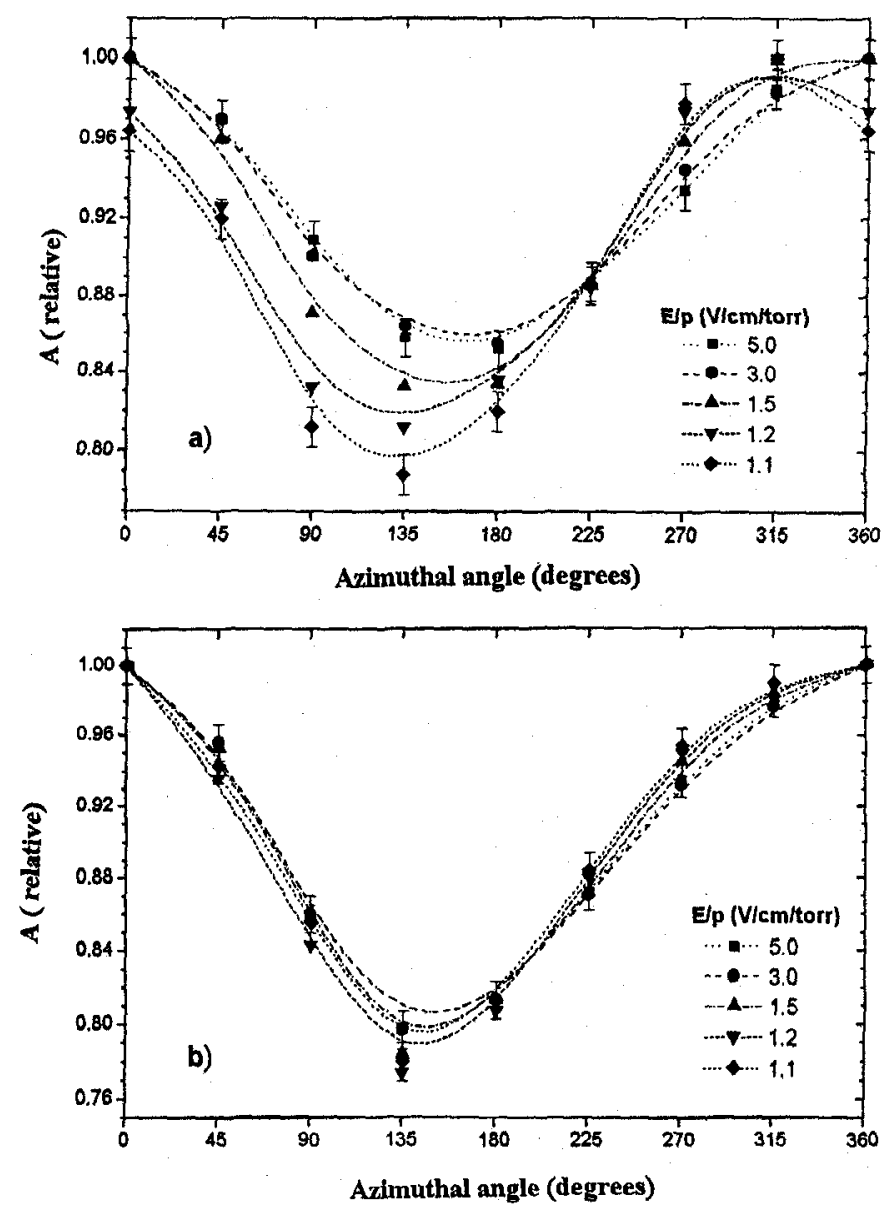

Figure 4: Relative centroid position, $A$, of the pulse-height distributions for $5.9-\mathrm{keV} \mathrm{x}$-rays as a function of azimuth of the $\mathrm{x}$-ray interaction point, at a fixed radius of $10 \mathrm{~mm}$, for several values of reduced electric field $E / p$ in the scintillation region. The $A$ values are normalized to the azimuthal direction $\phi=0$ (see text). Fig. 4a) for GPSC-1, Fig.4b) for GPSC-2. The curved lines are intended as guides to the eye.

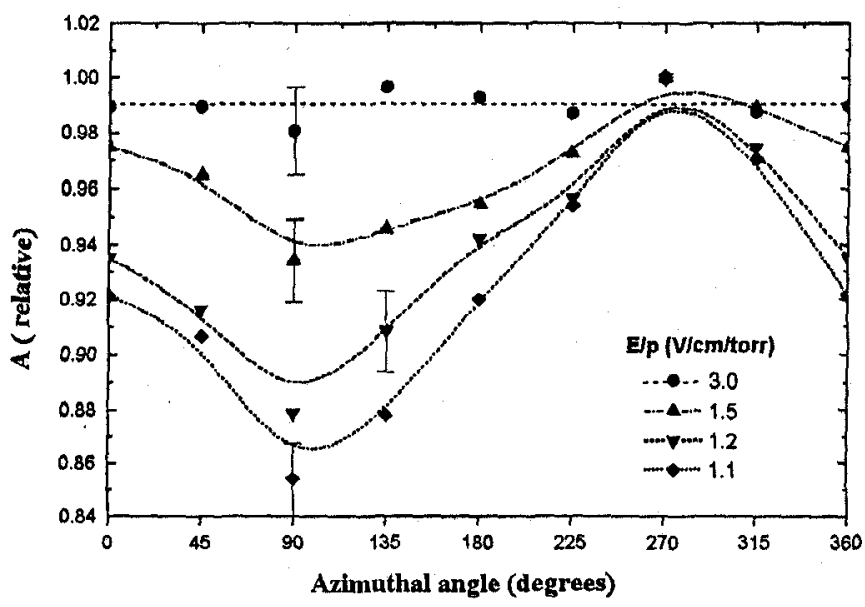

Figure 5: Normalized centroid-position, A, of the pulse-height distributions for $5.9-\mathrm{keV} \mathrm{x}$-rays as a function of azimuth of the $\mathrm{x}$-ray interaction point, at a fixed radius of $10 \mathrm{~mm}$, for several reduced electric field values, $E / p$, in the scintillation region. The $A$ values are normalized to those for $E / p=5.0 \mathrm{~V}-\mathrm{cm}^{-1}-\mathrm{torr}^{-1}$ (see text). The curved lines are intended as guides to the eye. 
misalignment display a sinusoidal trend with $\phi$. From Fig. 5 we can conclude that the direction of maximum tilt between grids is the $90 / 270$ degrees direction. Maximum variations in $\mathrm{A}(\phi)$ attributable to grid misalignment are approximately 15 , 12,7 and $2 \%$ for reduced electric fields of $1.1,1.2,1.5$ and $3.0 \mathrm{~V} \mathrm{~cm}^{-1}$ torr ${ }^{-1}$ in the scintillation region. This result is approximately a factor of two less than the prediction of Equation (2) for the lowest value of $\mathrm{E} / \mathrm{p}$. The magnitude of the effect is not as large as the $30 \%$ value predicted by Equation (2), due in part to the reduced dependence on $\mathrm{E} / \mathrm{p}$ near the scintillation threshold.

On the other hand, data in Figure $4 \mathrm{~b}$ for the well-aligned grids of GPSC-2demonstrate that there is little difference between the curves $A(\phi)$ for the different reduced electric fields in the scintillation region.

A more accurate fitting to the scintillation yield (see Figure 2) than Equation (2) should reproduce the measured results depicted in Figure 5. In fact, for the type of grid misalignment shown in Figure 3, and for a fixed radial distance, the reduced electric field in the scintillation region will depend on the azimuthal angle, $\phi$, due to the intergrid distance variation. Since the intergrid distance $X$ at each $\phi$ is known, the reduced electric field E/p can be obtained, to a good approximation, by $\mathrm{V} /(\mathrm{px})$, for small grid misalignments [12], where $\mathrm{V}$ is the voltage difference between the grids. Then the number of scintillation photons, $N$, produced by each electron along the field line between $\mathrm{Gl}$ and $\mathrm{G} 2$ can be given by

$$
N=x \frac{Y}{p}(E / p)
$$

where $\mathrm{Y} / \mathrm{p}$ is the reduced yield for the calculated reduced field value, $\mathrm{E} / \mathrm{p}$, as derived from Fig. 2 .

Assuming that grid misalignment is the only effect, the total number of scintillation photons produced as a function of $\phi$ for a fixed radius $R$ and normalized to the maximum value $\mathrm{N}_{1}$, is given by

$$
\frac{\mathrm{N}}{\mathrm{N}_{1}}=\frac{X \frac{Y}{p}}{X_{1} \frac{Y_{1}}{p}}=\frac{X \cdot Y(E / p)}{X_{1} Y_{1}\left(E_{1} / p\right)}
$$

For a given grid misalignment, $x(\phi)$ and $E(\phi) / p$, the relative variations in $N$ with $\phi$ can be calculated from Equation (4) if the values for $\mathrm{Y}(\mathrm{E} / \mathrm{p})$, absolute or relative values, are available (e.g. in Fig. 2).

In Figure 6 we depict the calculated relative variations of $\mathrm{N}$ as a function of the azimuthal angle for the GPSC-1 misalignment conditions at a radial distance of $10 \mathrm{~mm}$, and for several $E / p$ values in the scintillation region calculated at the detector axis. The 90/270 degrees direction was chosen to be the direction of maximum tilt between the grids. The results were obtained by fitting a polynomial to the values of $\mathrm{Y} / \mathrm{p}$ in Figure 2 and using those results in Equation (4). For improved accuracy, $E / p$ values above and below $2.5 \mathrm{~V} \mathrm{~cm}$ ${ }^{1}$ torr $^{-1}$ were fit with different polynomial functions. The results are in good agreement with those of Figure 5, measured directly in the GPSC-1.

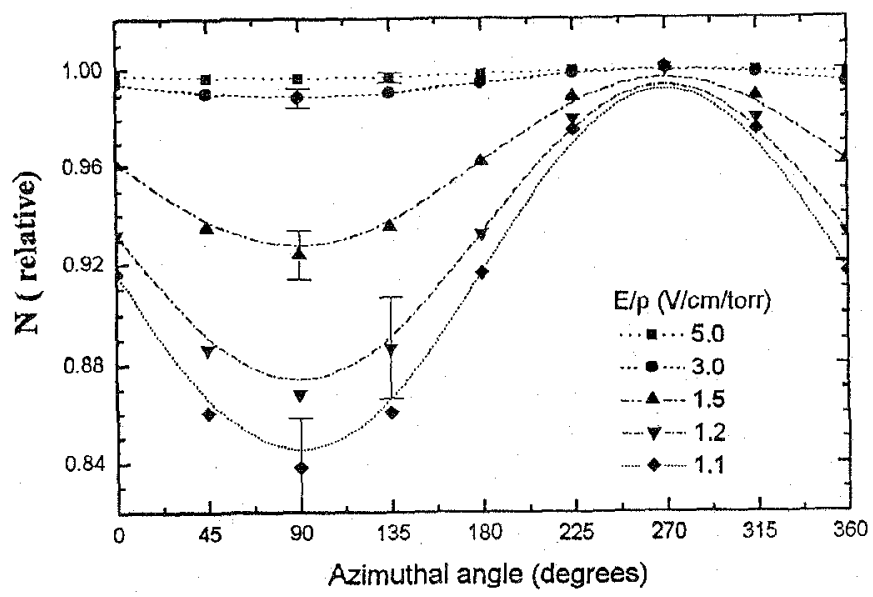

Figure 6: Relative variations in the total number of scintillation photons, $N$, produced as a function of azimuth of the $\mathrm{x}$-ray interaction point, at a fixed radius of $10 \mathrm{~mm}$, for the GPSC-1 misalignment conditions, and for several $\mathrm{E} / \mathrm{p}$ values (see text).The curved lines are intended as guides to the eye.

The errors for the relative scintillation light were estimated using the uncertainties of the experimental values for $\mathrm{Y} / \mathrm{p}$ in Figure 2. Relative uncertainties were less than $4 \%$ for $\mathrm{E} / \mathrm{p}$ above $1.0 \mathrm{~V} \mathrm{~cm}^{-1}$ torr $^{-1}$, less than $2 \%$ for $\mathrm{E} / \mathrm{p}$ above $1.3 \mathrm{~V} \mathrm{~cm}^{-1}$ torr ${ }^{-1}$, and less than $1 \%$ for $\mathrm{E} / \mathrm{p}$ above $4.5 \mathrm{~V} \mathrm{~cm}^{-1}$ torr ${ }^{-1}$.

\section{MONTE CARLO CALCULATIONS}

The experimental results were compared with the results of a Monte Carlo simulation program detailed in reference [11] and adapted for this purpose. This simulation accounts for diffusion of the primary electron cloud across the drift region, the solid angle of the PMT photocathode as viewed by the primary electrons along their scintillation path, and any reflections of the UV scintillation in the PMT window. A uniform quantum efficiency is assumed over the entire area of the photocathode.

For each azimuth, $\phi$, the Monte Carlo simulation program follows the history of seventy five $5.9 \mathrm{keV} x$-rays which generate a total of $2 \times 10^{4}$ electrons at a fixed radius, $r=10$ $\mathrm{mm}$. These electrons are allowed to drift and diffuse through the drift region [11]. In the scintillation region, the electrons are traced along the paths defined by the local electric field lines. Scintillation photons are generated at each successive inelastic collision whose position, $\mathrm{z}$, is defined using the average distance $\Delta z$ between two successive inelastic collisions [11], $z_{i+1}=z_{i}+\Delta z$. The $\Delta z$ values as a function of $\mathrm{E} / \mathrm{p}$ were obtained from a Monte Carlo simulation program [19]. The total number of UV photons produced in the 
scintillation region, $N_{p}$, and those collected by the PMT photocathode, $\mathrm{N}_{\mathrm{c}}$, are calculated. $\mathrm{N}_{\mathrm{c}}$ is assumed to be proportional to the centroid position, $A$, of the measured pulse-height distribution.

In Figure 7, the relative number of simulated UV photons collected by the photocathode is depicted as a function of the azimuthal angle for the GPSC-1 misalignment conditions at a radial distance of $10 \mathrm{~mm}$, for several values of $\mathrm{E} / \mathrm{p}$ calculated at the detector axis. Again, the 90/270 degrees direction was chosen to be the direction of maximum tilt between the grids. The Monte Carlo results are in good agreement with the experimental measurements. In addition, by calculating $\mathrm{N}_{\mathrm{p}}$ and $N_{c}$, the Monte Carlo simulations permit a separation of the variations due to solid angle effects from those due to scintillation photon production.

Monte Carlo errors are derived from uncertainties in the calculated values for the average distance between scintillation collisions, $\Delta z$, and are difficult to assess. To estimate these errors, another independent set of $\Delta z$ values were obtained from the reduced scintillation yield data of Figure 2. Absolute values for the $\Delta \mathrm{z}$ parameter were obtained by normalizing the relative values of Figure 2 to the absolute $\mathrm{Y} / \mathrm{p}$ values obtained by the Monte Carlo program of [19] that were presented in [14], for large values of $E / p$. The relative difference between the $\Delta z$ values obtained with both sets of data could be as great as $20 \%$ for the same value of $E / p$. However, these $\Delta z$ differences are reflected in pulse amplitude variations of about $3 \%$ for $\mathrm{E} / \mathrm{p}=1.1 \mathrm{~V} \mathrm{~cm}^{-1}$ torr ${ }^{-1}$, to less than $1 \%$ for $E / p=5 \mathrm{~V} \mathrm{~cm}^{-1}$ torr $^{-1}$ as determined by the relative number of UV scintillation photons collected, $\mathrm{N}_{\mathrm{c}}$.

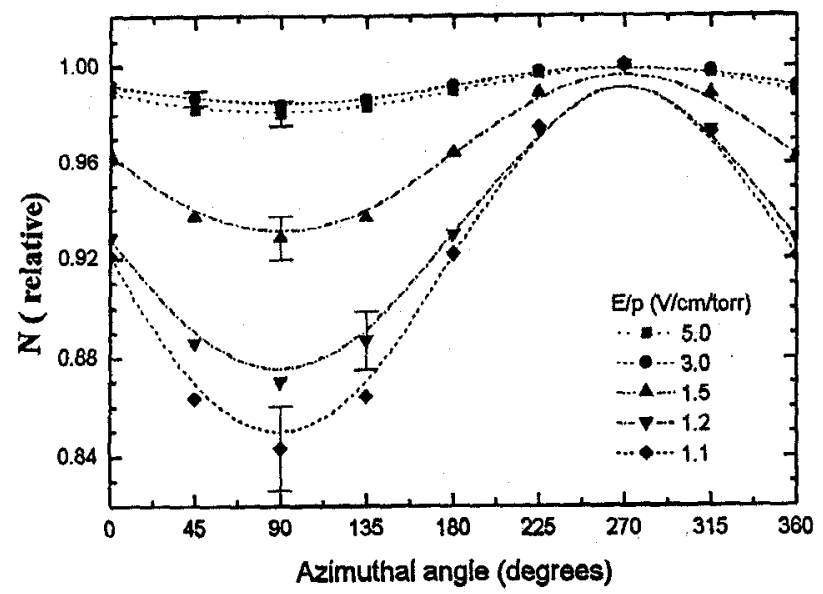

Figure 7: Relative number of simulated scintillation photons collected by the photocathode, $N_{c}$ as a function of azimuth for the GPSC-1 misalignment conditions at a fixed radius of $10 \mathrm{~mm}$, for several values of $E / p$ (see text). The curved lines are intended as guides to the eye.

\section{ANALYSIS AND DISCUSSION}

For the purposes of analysis and discussion, we define the maximum relative variation in the centroid position of the pulse-height distributions, $A(\phi)$, at radial distances of $10 \mathrm{~mm}$ for the $3 \%$ grid misalignment geometry of GPSC-1, as:

$$
\frac{\Delta \mathrm{A}}{\mathrm{A}}=\frac{A_{270}-A_{90}}{A_{270}}
$$

Similarly, we represent the maximum relative variations in the reduced total number of scintillation photons $\mathrm{N}_{\mathrm{p}}(\phi)$ as:

$$
\frac{\Delta \mathrm{N}}{\mathrm{N}}=\frac{N_{270}-N_{90}}{N_{270}}
$$

In Table I we present the results of $\triangle \mathrm{A} / \mathrm{A}$ as a function of the reduced electric field in the scintillation region for the experimental measurements in GPSC- 1 and the Monte Carlo simulation results which include solid angle effects.

In Table II we present the results of $\Delta \mathrm{N} / \mathrm{N}$, as a function of the reduced electric field in the scintillation region for the linear and polynomial fits to the data of Figure 2, and for the Monte Carlo simulation, for which solid angle effects are not included.

Table 1

Maximum relative variations (\%) $\triangle \mathrm{A} / \mathrm{A}$ as a function of the reduced electric field in the scintillation region for experimental measurements in GPSC-1 and for the Monte Carlo simulation.

\begin{tabular}{ccc}
\hline $\begin{array}{c}\mathrm{E} / \mathrm{p} \\
\left(\mathrm{Vcm}^{-1} \text { Torr }^{-1}\right)\end{array}$ & Monte Carlo & $\begin{array}{c}\text { Exp. } \\
\text { (GPSC-1) }\end{array}$ \\
\hline 5.0 & $1.6 \pm 0.5$ & - \\
3.0 & $1.8 \pm 0.3$ & $2 \pm 2$ \\
1.5 & $7.9 \pm 0.9$ & $7 \pm 2$ \\
1.2 & $13.1 \pm 1.2$ & $12 \pm 2$ \\
1.1 & $16.9 \pm 1.7$ & $15 \pm 2$ \\
\hline
\end{tabular}

"Value obtained for a 10-mm deep scintillation region.

+Values normalized to $E / \mathrm{p}=5.0 \mathrm{~V}-\mathrm{cm}^{-1}-$ torr $^{-1}$.

Table 2

Maximum relative variations (\%) $\Delta N / N$ as a function of the reduced electric field in the scintillation region for the linear and polynomial fits to the data of Figure 2 and for the Monte Carlo simulations

\begin{tabular}{cccc}
\hline $\begin{array}{c}\mathrm{E} / \mathrm{p} \\
\left(\mathrm{Vcm}^{-1} \text { Torr }^{-1}\right)\end{array}$ & $\begin{array}{c}\text { linear fit } \\
(\mathrm{Eq} .(2))\end{array}$ & $\begin{array}{c}\text { polynomial } \\
\text { fit }\end{array}$ & Monte Carlo \\
\hline 5.0 & 0.8 & $0.4 \pm 0.2$ & $0.7 \pm 0.4$ \\
3.0 & 1.5 & $1.2 \pm 0.4$ & $1.0 \pm 0.4$ \\
1.5 & 6 & $8 \pm 1$ & $7.2 \pm 0.8$ \\
1.2 & 15 & $13 \pm 2$ & $12.6 \pm 1.1$ \\
1.1 & 30 & $16 \pm 2$ & $16.4 \pm 1.7$ \\
\hline
\end{tabular}

"Value obtained for a 10-mm deep scintillation region.

Good agreement was found between the experimental results obtained directly from the GPSC-1 measurements, from the polynomial fit to the relative reduced 
electroluminescence yield values of Fig.2, and from the Monte Carlo simulation program. The linear approximation leads to results that are significantly higher than those obtained with a more accurate fitting for $E / p$ values approaching the scintillation threshold.

The results of the Monte Carlo simulation indicate that the contribution of the solid angle variation is not negligible and becomes more significant with increasing values of $\mathrm{E} / \mathrm{p}$. In Table 2 , the $\Delta N / N$ include only the variation in scintillation yield while the Monte Carlo results for $\triangle \mathrm{A} / \mathrm{A}$ include both the variation in the scintillation yield and the effects of the solid angle. For low values of $E / p$, the variation in scintillation yield is dominant but, as $\mathrm{E} / \mathrm{p}$ increases the variation in the scintillation yield decreases while the variation due to the solid angle does not change significantly. For high values of $\mathrm{E} / \mathrm{p}$, the variation due to the solid angle becomes as important as the scintillation yield variation. In fact; for $\mathrm{E} / \mathrm{p}=5.0 \mathrm{~V} \mathrm{~cm}^{-1}$ torr ${ }^{-1}$, although the maximum variation in the total scintillation due to grid misalignment is about $0.7 \%$ (and even less if the results of the polynomial fit to the yield is used), the variation for the number of scintillation photons collected by the PMT is about $1.6 \%$.

\section{VII, CONCLUSTONS}

A detailed exposition of the effects of grid misalignment in the scintillation region of a gas proportional scintillation counter has been presented. The results indicate that the simplifying assumption of a linear dependence of scintillation light output on the reduced electric field is inaccurate.

When a more accurate and detailed representation of the grid misalignment processes that affect pulse-height distributions are considered, good experimental agreement is found between the results measured directly in a misaligned GPSC and the relative reduced scintillation yield values measured as a function of the reduced field, $\mathrm{E} / \mathrm{p}$.

It has also been demonstrated that solid-angle effects have to be considered when evaluating the effects of grid misalignment for the reduced field values in the scintillation region of an optimized GPSC. Misalignments as gross as 3\% will have a larger effect, by a factor of about two, on pulseheight amplitude variations than the prediction of Equation (2). Although this effect is still negligible compared to statistical fluctuations in the number of primary electrons nominally produced in the absorption of $x$-rays in the $\mathrm{keV}$ energy region, the effect is more dramatic for reduced fields closer to the scintillation threshold $\left(\sim 1.1 \mathrm{~V} \mathrm{~cm}^{-1} \operatorname{torr}^{-1}\right)$. In that case, pulse-height amplitude variations as large as $16 \%$ are possible, a factor of two smaller than what is predicted by Equation (2), although it is unlikely that a GPSC would be operated under those unfavorable conditions.

\section{ACKNOWLEDGMENTS}

Thanks are due to F.P. Santos, P.J.B.M. Rachinhas, and F.I.G. Borges for providing valuable data. This work was funded bv Junta Nacional de Investigação Científica (Lisboa) through 'esearch project Stride No. CERN/C/FIS/1085/96.

\section{REFERENCES}

[1] A.J.P.L. Policarpo, "The Gas Proportional Scintillation Counter", Space Sci. Instrum. Vol. 3, p. 77, 1977.

[2] A. Smith and M. Badvaz, "Gas proportional scintillation counters for x-ray synchrotron applications", Rev. Sci. Instr. Vol. 63(1), p. 689, 1992.

[3] V.P. Varvaritsa, I.V. Vikulov, V.V. Ivashov, M.A. Panov, V.I. Filatoy and K.I. Schekin, "Proportional Scintillation detectors", Instr. and Experim. Techniques Vol. 35, p. 745, 1992

[4] J.M.F. dos Santos, A.C.S.S.M. Bento and C.A.N Conde, "The performance of the curved grid gas proportional scintillation counter in x-ray spectrometry", Nucl. Instr. \& Meth. in Phys. Res. A Vol. 337, p 14271994.

[5] J.M.F. dos Santos, J.F.C.A.Veloso, R.E.Morgado, and C.A.N Conde, "The performance of a compact gas proportional scintillation counter for hard x-ray spectrometry", Nucl. Instr. \& Meth. in Phys. Res. A Vol.353, p. 195, 1994.

[6] A. Niemela, H. Sipila and V.I. Iyanov, "High -Resolution p-i-n CdTe and CdZnTeX-Ray Detectors with Cooling and Rise-Time Discrimination", IEEE Trans. Nucl. Sci. Vol. 43(3), p. 1476, 1996.

[7] J.S. Iwanczyk, B.E. Patt, Y.J. Wang, M. Croft, Z. Kalman and W. Mayo, "High Efficiency $\mathrm{CsI}(\mathrm{TI}) / \mathrm{HgI}_{2}$ Gamma ray Spectrometers", IEEE Trans. Nucl. Sci. Vol. 42(4), p. $601,1995$.

[8] A. Peacock, R.D. Andresen, E.A. Leiman, A.E. Long and G. Manzo, "Performance characteristics of a gas scintillation spectrometer for x-ray astronomy", Nucl. Instr. Meth. Vol. 169, p. 613, 1980.

[9] D.G. Simons, P.A.J. de Korte and J.A.M. Bleeker, "Energy resolution limitations in a gas scintillation proportional counter, SPIE Symp. on X-Ray Instr. in Astronomy Vol. 597, p. 190, 1985.

[10] J.M.F. dos Santos, A.C.S.S.M. Bento and C.A.N. Conde, "The effect of the photomultiplier nonuniformity on the performance of gas proportional scintillation counters", Nucl. Instr. Meth. A Vol. 321, p. 238, 1992.

[11] J.M.F. dos Santos, A.C.S.S.M. Bento and C.A.N Conde, "The dependence of energy resolution of gas proportional scintillation counters on the scintillation-tophotomultiplier distance", IEEE Trans. Nucl. Sci. Vol.39(4), p. 541, 1985.

[12] C.A.N. Conde, L.R. Ferreira and M.F.A. Ferreira, "The secondary scintillation output of xenon in a uniform field gas proportional scintillation counter", IEEE Trans. $\mathrm{NuCl}$. Sci. Vol. 24, p. $221,1977$. 
[13] F. Favata, A. Smith, M. Bavdaz and T.Z. Kowalski, "Light yield as a function of gas pressure and electric field in gas proportional scintillation counters", Nucl. Instr. Meth. A Vol. 294, p. 595, 1990.

[14] F.P. Santos, T.H.V.T. Dias, A.D. Stauffer and C.A.N. Conde, "Three-dimensional Monte Carlo calculation of the VUV electroluminescence and other electron transport parameters in xenon", J. Phys. D, Vol. 27, p. 42, 1993.

[15] J.M.F. dos Santos, T.H.V.T. Dias, S.D.A.R. Cortes and C.A.N. Conde, "Novel techniques for designing gas proportional scintillation counters for x-ray spectrometry", Nucl. Instr. Meth. A, Vol. 280, p. 288, 1989.
[16] J.M.F. dos Santos, A.C.S.S.M. Bento and C.A.N Conde, "Simple inexpensive gas proportional scintillation counter for $\mathrm{x}$-ray fluorescence analysis", X-Ray Spectrometry, Vol. 22, p. 328, 1993.

[17] P.R. Bevington, Data reduction and error analysis for the physical sciences, pp. 208-214, McGraw Hill, New York, 1969.

[18] J.M.F. dos Santos, J.F.C.A. Veloso, J.A.M. Lopes and R.E. Morgado, "A simple method to improve the spatial uniformity of venetian-blind photomultiplier tubes", IEEE Trans. Nucl. Sci., Vol.43(3), p. 1335, 1995.

[19] T.H.V.T.Dias, F.P.Santos, A.D.Stauffer and C.A.N Conde, "A Monte Carlo simulation of x-ray absorption and electron drift in gaseous xenon", Phys. Rev. A, Vol. 48, p. 2887, 1993. 